\title{
ATUAÇÃO DA FISIOTERAPIA ONCOLÓGICA PEDIÁTRICA ATRAVÉS DE CUIDADOS PALIATIVOS
}

\section{PERFORMANCE OF PEDIATRIC ONCOLOGICAL PHYSIOTHERAPY THROUGH PALLIATIVE CARE}

\author{
Juliana Santos Sosta, \\ Estudante do 9º período de fisioterapia da Faculdade \\ Presidente Antônio Carlos deTeófilo Otoni. Brasil. \\ E-mail: julianasc97@hotmail.com
}

Lorena Slves Colen, Estudante do 9ำ período de fisioterapia da Faculdade Presidente Antônio Carlos de Teófilo Otoni.Brasil. E-mail: loreninhacolen@hotmail.com

Rejane Goecking b. Pereira

Especialista em Fisioterapia Neurológica adulto e infantil, Especialista em Urgência e Emergência pelo Hospital Sírio Libanês, Especialista em Neonatolotia, RT do serviço de Fisioterapia da Unimed, Professora de Fisioterapia na Faculdade Presidente Antônio Carlos de Teófilo Otoni, Brasil. E-mail: rejanegoecking@hotmail.com

\section{Resumo}

O presente trabalho visa uma interligação entre a oncologia, cuidados paliativos e a fisioterapia oncológica pediátrica, apresentando uma proposta de interdisciplinaridade entre áreas de conhecimento de forma contextualizada. $O$ trabalho aborda os conceitos de câncer, câncer infantil, cuidados paliativos e a aplicação de técnicas fisioterápicas a fim de preservar e ou restaurar a integridade funcional de áreas específicas do corpo, que sofreram ação de células cancerígenas, um estudo que pode vir a colaborar com a aprendizagem dos conteúdos em pauta. Tem como objetivo desenvolver uma perspectiva de ensino e aprendizagem que possa promover a interação entre os conteúdos e tornar os fisioterapeutas profissionais multifacetados. Espera-se com este trabalho propor uma didática 
diferenciada dentro e fora da sala de aula, que contribua com o desenvolvimento do interesse dos alunos para a aprendizagem dos conteúdos, de forma que possam apreende-los e aplica-los no combate e prevenção ao câncer.

Palavras-chave: Cuidados Paliativos, Fisioterapia Oncológica Pediátrica, Câncer.

\section{Abstract}

The present work aims at an interconnection between oncology, palliative care and pediatric oncological physiotherapy, presenting an interdisciplinary proposal between areas of knowledge in a contextualized way. The work addresses the concepts of cancer, childhood cancer, palliative care and the application of physiotherapy techniques in order to preserve and or restore the functional integrity of specific areas of the body, which have been affected by cancer cells, a study that may come to collaborate with learning the content on the agenda. It aims to develop a teaching and learning perspective that can promote interaction between content and make physical therapists multifaceted. It is expected with this work to propose a different didactics inside and outside the classroom, which contributes to the development of the students' interest in learning the contents, so that they can apprehend them and apply them in the fight and prevention of cancer. The studies found show that physiotherapy has an important role in palliative care, however there is a need for a greater offer and implementation of this treatment in health services.

Keywords: Palliative Care, Pediatric Oncological Physiotherapy, Câncer.

\section{Introdução}

A Fisioterapia atua na estimulação de regiões especificas do corpo, a fim de promover uma melhor organização sensitiva e motora aos pacientes. Essa prática tem sido amplamente utilizada no combate aos efeitos do tratamento contra o câncer. A Fisioterapia Oncológica voltada a crianças desempenha um importante papel na minimização dos efeitos adversos do tratamento ao câncer, tendo em vista que se trata de uma doença crônica degenerativa, que se define pelo crescimento desordenado de células cancerosas que invadem tecidos e ou órgãos, podendo se espalhar por outras regiões do corpo, diferentes do seu local de origem (metástase).

Os Cuidados Paliativos visam à qualidade de vida dos pacientes oncológicos infantis, bem como a de seus familiares, em situações em que a condição ameace a continuidade da vida, prevenindo e ou amenizando o sofrimento do paciente. A identificação precoce, avaliação e tratamento da dor e de sintomas físicos, psíquicos e sociais também são parte do tratamento. 
De acordo com análise e interpretação dos dados do Instituto Nacional de Câncer (INCA) constata-se que a incidência de câncer tem aumentado ao longo dos anos, chegando aos 17.000 .000 (dezessete milhões) de novos casos no ano de 2018, sendo os mais recorrentes em crianças e adolescentes, as leucemias, correspondendo a 33\% (trinta e três por cento) desse total. Com base nestes dados observa-se que se deve aprofundar os conhecimentos, preenchendo as lacunas, de forma a nos informar ao máximo, o que confere mais segurança no diagnóstico, tratamento e cuidados no processo terminal.

O trabalho pretende inserir e ou incorporar às discussões e estudos o aperfeiçoamento das práticas já existentes e inserção de novas práticas.

\subsection{Objetivos}

Compreender os benefícios da prática profissional do fisioterapeuta nos cuidados paliativos como forma de amenizar o sofrimento psíquico, físico e familiar dos pacientes oncológicos infantis, proporcionando-lhes alívio nas dores e uma melhor qualidade de vida durante o tratamento.

\section{Revisão Literária}

\subsection{Câncer}

No ano de 2018, surgiram 17.000.000 (dezessete milhões) de novos casos de câncer no mundo, sendo 559.371 (quinhentos e cinquenta e nove mil e trezentos e setenta e um) deles diagnosticados no Brasil, dados do CÂNCER RESEARCH UK E AGÊNCIA INTERNACIONAL PARA PESQUISA DO CÂNCER. Nesse mesmo ano, mais de 9.600 .000 (nove milhões e seiscentos mil) pessoas vieram a óbito no mundo vítimas de câncer, sendo 243.588 (duzentos e quarenta e três mil e quinhentos e oitenta e oito) no Brasil.

O diagnóstico deve sempre ser feito o mais cedo possível a fim de mitigar os malefícios dessa condição, afinal o diagnóstico não é mais tido como uma sentença de morte, já que os tratamentos e pesquisas nessa esfera avançaram bastante ao longo dos anos. 
Câncer, segundo INCA (2007, p.8) é dominado como um complexo conjunto de mais de 100 (cem) doenças, de um mesmo grupo, que afetam seres humanos e animais, caracterizadas pela capacidade de atacar e se infiltrar em células sadias, descoberta por Hipócrates, o pai da Medicina.

De acordo com estudos de Muller (2011, p.57), as células cancerígenas têm algumas particularidades, sendo uma delas a capacidade de se passar por células saudáveis, "driblando" assim a função de proteção do sistema imunológico. As células saudáveis morrem conforme o seu organismo não as solicite, o mesmo não ocorre com células cancerosas. (Muller, 2011, p.57).

O Câncer se dá através de três etapas, mapeadas pela ciência, a primeira delas ou Estágio de Iniciação, acontece quando as células sofrem a ação de um agente cancerígeno, que as modifica geneticamente. Nesse estágio o câncer não é detectável e as células preparam-se para receber a ação de um segundo grupo de agentes. Na segunda etapa ou Estágio de Promoção, esse grupo de células iniciadas, já geneticamente alteradas sofre a ação do segundo grupo de agentes e o contato constante com o mesmo ocasiona um processo lento e gradual que dá origem a células cancerígenas. (Associação Brasileira do Câncer [ABC], 2007).

A última, mas não menos importante é a etapa três ou Estágio de Progressão, nesse nível as células cancerosas multiplicam-se de forma descontrolada, sendo esse estágio irreversível, pois nele o câncer já está instalado e evoluindo, é nele que ocorre os mais de 200 (duzentos) tipos de tumores, que surgem em qualquer lugar do corpo e que podem ser detectados clinicamente, a exemplo temos os linfomas e leucemias.

Ainda, segundo a ABC (2007); INCA (2007) destaca-se alguns de tipos de câncer que acometem os organismos:

a) As leucemias são o acúmulo de células cancerígenas na medula óssea (líquido gelatinoso localizado no interior dos ossos). Essas substituem as células saudáveis, prejudicando a produção de glóbulos vermelhos, brancos e plaquetas.

b) Os linfomas surgem no sistema linfático (principal meio de defesa do nosso organismo) atingindo as glândulas de todo o corpo. 
c) O carcinoma afeta as células epiteliais (pele e ou tecidos que revestem nossos órgãos). Sarcoma são tumores raros que afetam os ossos, músculos, células de gordura e cartilagens.

d) Tumores do Sistema Nervoso Central (ou tumores SNC) são agressivos

em demasia, afetam o cérebro e as células de glia, que existem para dar suporte para os neurônios, sendo o segundo mais comum entre indivíduos de até 18 (dezoito) anos, dados do SALK INSTITUTE.

Considerando que os tumores podem ser benignos, isto é, aqueles que se desenvolvem lentamente e não afetam células saudáveis e a depender de sua localização não afetam positiva ou negativamente a vida do paciente, e malignos, onde as células multiplicam-se descontroladamente, afetando células saudáveis e por consequência a vida do paciente. Há ainda outra condição relacionada às células cancerosas, ela se dá quando essas, "viajam" através dos vasos sanguíneos afetando outras células e tecidos, manifestando-se em regiões diferentes do seu local de origem, a esse processo damos o nome de METÁSTASE. (ABC, 2007; INCA, 2007; Núcleo de Apoio à Criança com Câncer [NACC], 2007

Atualmente existem maneiras de combate ao câncer. Uma delas é a Cirurgia, onde há extração da massa de células malignas, isto é, literalmente a retirada do tumor, o impedindo de se espalhar.

Quando a retirada completa não é possível, é realizada a retirada de uma parte, que serve de base para estudos que ajudarão no tratamento da doença com medicamentos específicos. A mais conhecida é a Quimioterapia, que faz uso de medicamentos que se misturam ao sangue e são levados a todas as partes do corpo, o que pode destruir as células cancerígenas ou impedir sua multiplicação. Esse tratamento traz, na maioria das vezes, efeitos colaterais, como queda de cabelo, fadiga, perda de apetite, enjoo, anemia, infecções e hemorragias. (BRASIL, 2005, p.35)

A Radioterapia "bombardeia" o corpo com radiação, o que ajuda a controlar o tamanho do tumor ou exterminar parte das células cancerígenas. Os efeitos colaterais afetam normalmente apenas a área tratada e se manifesta através de alergias, edemas, inchaço, prurido e feridas na pele. 
Nos dias de hoje, descoberta mais recente da ciência, a Imunoterapia consiste em utilizar drogas que ensinam o corpo a detectar células cancerosas e a combatê-las por conta própria. Nessa modalidade temos um tratamento, disponível em poucos países, que usa células geneticamente modificadas para combater essa condição. Inclusive, no Brasil, a USP e FAPESP desenvolveram uma versão desse tratamento, usando apenas de tecnologia nacional e a testaram em uma paciente, que respondeu bem ao tratamento.

Entretanto, ressalte-se que esta não é a tão esperada cura para o câncer, já que o tratamento não funciona da mesma forma para todos os indivíduos. Ainda neste âmbito, temos a Terapia Alvo, um estudo para o uso de drogas que funcionem como uma espécie de "chave" para abertura de uma fechadura especifica, atua no combate a estruturas presentes nas células cancerosas, reduzindo o dano que elas podem causar, em outras palavras um ataque benéfico a nível molecular.

\subsection{Considerações sobre o câncer infantil}

\subsubsection{O Câncer Infantil}

O câncer infantil, é uma incógnita em relação aos fatores de risco que podem desencadear, ou ativar a doença, sendo que nos cânceres adultos, são influenciados por fatores ambientais, hábitos alimentares, estilos de vida e aspectos emocionais. Nesse sentido, ressalta-se que o diagnóstico precoce é de suma importância para que essa doença seja tratada.

Ressalta-se, que existem sintomas de doenças cotidianas comuns que podem ser relacionadas a sintomas do câncer infantil, ocasionando assim uma certa confusão no diagnóstico das crianças que desenvolveram o câncer infantil. Assim como consequência há um certo retardamento da detecção precoce da doença, e neste sentindo, os pais das crianças devem ficar atentos a qualquer tipo de sintomas e no surgimento de alguns deles, e com isso a família deve procurar um especialista para que seja feito o diagnóstico correto.

Assim sendo, quando mais rápido for diagnosticado o câncer infantil, fica mais fácil de impedir que a doença se agrave e com isso o prognóstico seja ruim. Um ponto que se deve levar em consideração é que, não existem exames 
preventivos feitos rotineiramente em crianças para detecção do câncer, ao contrário do que acontece com os adultos. Com isso a importância da avaliação periódica de um pediatra durante toda a infância (COSTA, et. al 2006)

Existem muitos procedimentos utilizados na detecção dos cânceres. Entre os mais comuns e conhecidos estão: biópsia, punção, ultrassonografia, tomografia computadorizada, ressonância magnética, hemograma, mielograma (exame da medula óssea), entre outros.

Diversos fatores combinados, determinam se o prognóstico de uma criança com câncer será bom ou ruim, tais como: tipo de câncer, sua classificação (benigno ou maligno) e o estágio em que ele se encontra, sendo que esses fatores são determinados pela precocidade do diagnóstico, assim observa-se a importância de uma descoberta precoce para um melhor prognóstico para criança com câncer.

Segundo o INCA (Instituto Nacional de Câncer), a incidência do câncer infantil aponta que a taxa média ajustada por idade varia entre 76 e 231, sendo a maior observada em Belém do Pará e a menor em Goiânia. Tanto no Brasil quanto no mundo, o predomínio maior de câncer é a leucemia em crianças e adolescentes.

É a maior causa de morte em crianças, mas quando há um diagnóstico precoce, as chances de cura podem chegar até a 70\%, segundo o ministério da saúde. Os tipos de tumores mais comuns na infância e adolescência são as leucemias que afeta diretamente os glóbulos brancos e os linfomas que ataca o sistema linfático.

\subsubsection{Diagnóstico}

As causas do câncer infantil são múltiplas e ainda desconhecidas, porém há fatores que indicam a questão genética atrelada a possíveis causas como alimentação inadequada, exposição direta ao sol e alguns vírus que podem causar a leucemia.

Para os familiares não é nada fácil receber e aceitar o diagnóstico da doença, sobretudo quando pesa a dor que os dias da criança serão abreviados pela invasão de células cancerígenas atuando em determinada área do organismo.

Para o correto diagnóstico é necessário a solicitação de exames específicos pelo médico, como AFP, CA125 e BTA, marcadores tumorais que detectam células 
cancerígenas, além de um laboratório de confiança. Pode ser realizado também a biópsia, um pequeno procedimento cirúrgico para recolher amostra do tecido para ser analisado em laboratório.

\subsubsection{Sinais e sintomas}

Os pais devem estar sempre alerta a qualquer reclamação dos filhos, investigar todo e qualquer sintoma. Pois quanto mais rápido for feito o diagnóstico, maior é a possibilidade de cura.

As pessoas cuidam dos seus sintomas e ignoram suas doenças, (VENCY) os sintomas nunca devem ser ignorados, pois o câncer é uma doença crônica com difícil diagnóstico precoce, e muitas vezes os sintomas podem ser confundidos com outros males comuns na infância.

Os sintomas como dor, inchaço sem trauma, sangramentos ou hematomas, febre, perda de peso sem causa aparente, vômitos, e mudanças de comportamento na criança, devem ser observados pelos pais o quanto antes, para uma melhor probabilidade de cura.

A leucemia é um dos tipos de câncer mais comuns da infância, afeta diretamente os glóbulos brancos do sangue, e seus sintomas em particular, são dor nos ossos e nas articulações, sangramentos, fadiga, fraqueza, febre e palidez.

\section{3 fisioterapia oncológica}

É uma área que tem sido muito estudada e pesquisada, e tem alcançado nos últimos anos desde a sua concepção grande relevância, mas no geral ainda requer reconhecimento, pois tem locais que infelizmente o fisioterapeuta não faz parte da equipe no tratamento do paciente com câncer.. Trata-se de uma especialidade cujo objetivo é preservar, desenvolver e restaurar a integridade de órgãos e sistemas do paciente, prevenir e amenizar distúrbios causados pelo tratamento oncológico. (PORTO et al., 2012, p.233)

Atua no pre e no pós-operatório, assim como durante os tratamentos de quimioterapia e radioterapia. Sua principal meta é mostrar ao paciente a 
necessidade de retomar suas atividades de vida diária e oferecer condições para isso.

O foco para o tratamento do paciente com câncer deixa de ser somente a cura e o controle da doença. A fisioterapia colabora ativamente na manutenção da qualidade de vida, desde o diagnóstico até o final do tratamento. Reabilita, condiciona e adapta o paciente à sua nova condição. (COSTA et al., 2007, p.78)

Nesse sentido observa-se que a especialidade é indicada para todas as pessoas que tenham recebido o diagnóstico da doença. Independentemente do sexo, da idade ou do tipo de câncer. Sabe-se que tanto o tratamento cirúrgico quanto o clínico (quimioterapia e radioterapia) podem trazer algumas limitações físicas, funcionais e emocionais ao paciente. Esses fatores impedem ou prejudicam algumas atividades da sua vida diária.

Segundo PESSINI; BERTACHINI, ( 2004, p.15), mostra que a fisioterapia tem desempenha um papel importante nos cuidados paliativos. Os recursos e habilidades utilizados por fisioterapeutas através dos conhecimentos adquiridos são importantes e úteis para os cuidados paliativos, pois proporcionam positivamente bons resultados para a equipe de saúde e seus pacientes, além de corroborar com a atuação familiar e sua ação colaboradora, e de forma integrada em atendimentos aos pacientes oncológicos no geral (GUTIERREZ, 2001, p.47).

\subsubsection{Fisioterapia Oncológica Pediátrica}

Quando criança, vive-se momentos de interações marcantes, tanto no âmbito familiar quanto em relações sociais com um todo, momento em que o indivíduo constrói sua relação tanto em si quanto com o mundo externo e a partir daí adquire uma estrutura de personalidade que vai ser a base para todas as suas experiências futuras.

Nesse contexto, quando se surpreendido com uma doença, no caso o Câncer, que é um evento inesperado e indesejado, e dependendo do tipo e da precocidade do diagnóstico, pode causar sequelas físicas e psíquicas que serão marcantes para a criança. Sendo que todo seu cotidiano é alterado resultado das limitações que a doença e tratamento impõem. 
E nesse sentido o papel da família é de suma importância. Segundo Dávila (2006) ressalta que nesse contexto, os pais são os primeiros a necessitarem de auxilio e ajuda, isso acontece, pois, a criança não conhecimento sobre a doença, e assim os pais acabam por transmitir os sentimentos provados pela descoberta do diagnóstico da doença. Assim uma família bem embasada, recebendo orientações adequadas, saberão lidar de maneira mais tranquila com a doença, e consequentemente estará mais pronta para assumir e procurar deixar os efeitos da doença menos prejudiciais para seus filhos.

Segundo o autor, as crianças começam a perceber a doença, no momento em que algumas rotinas do dia a dia em sua vida, muda de forma, que fica mais limitada, pois com os efeitos do tratamento, condiciona a criança uma certa indisposição, não podendo realizar as atividades que costumava fazer anteriormente.

\subsubsection{Cuidados Paliativos na Fisioterapia Oncológica Infantil}

A fisioterapia em cuidados paliativos visa a qualidade de vida em pacientes com doença avançada ou em progressão desta, por meio de condutas que reabilitem funcionalmente o paciente, bem como auxilia o cuidador a lidar com o avanço rápido da enfermidade. (INCA, 2018, p.16)

O conceito de cuidados paliativos, segundo Melo et al. (2013, p.76):

[...]teve origem no movimento hospice (hospitalidade), originado por Cecily Saunders e seus colegas, em 1950, disseminando pelo mundo uma nova filosofia sobre o cuidar, e não só curar, focado no paciente até o final de sua vida. A partir daí um novo campo foi criado, o da medicina paliativa, incorporando a essa filosofia, equipes de saúde especializadas no controle da dor e no alívio dos sintomas. À medida que a doença progride maior é a necessidade de cuidados paliativos, o que os tornam quase que exclusivos ao final da vida.

Müller, Scortegagna e Moussalle (2011, p.56), com a sua pesquisa acrescenta a ideia de Melo et al. (2013), ressaltando que os cuidados paliativos devem ser tratados em sua totalidade, isto é, a fisioterapia leva em consideração 
vários aspectos os psicológicos, os psicossociais, e os espirituais, assim estimula o paciente através das conversas, ouvindo-o atentamente, procurar da toda a atenção em todas as dimensões e com o apoio familiar.

Ainda segundo Melo et. Al (2013, p.65), os cuidados paliativos surgiram para suprir as necessidades específicas de pacientes sem possibilidades terapêuticas curativas. Sua inserção no atendimento de pacientes oncológicos é de extrema importância, sendo uma necessidade crescente nos hospitais e instituições de saúde.

Para Gutierrez (2001,p.47), admitir que se esgotaram os recursos para 0 resgate de uma cura e que o paciente se encaminha para o fim da vida, não significa que não há mais o que fazer. Ao contrário, abre-se uma ampla gama de condutas que podem ser oferecidas ao paciente e sua família. O papel da fisioterapia, neste contexto, foi descrito, no final de 1970, em vários livros e artigos científicos, nos quais a fisioterapia era descrita em diversos capítulos, e onde se referia a importância da equipe multidisciplinar nos cuidados paliativos (Laakso, 2006, p.35).

Cuidados paliativos pediátricos forma definidos em 1998. Como a assistência prestada ao paciente com doença crônica e/ou ameaçadora da vida. (Laakso, 2006, p. 35). O autor ressalta, que devem ser iniciados no diagnóstico, independentemente do tratamento da doença de base. Sendo que esses cuidados devem trabalhados de forma que envolvam uma equipe com vários profissionais (multiprofissional) que dão todo o suporte tanto físico, quanto emocional, espiritual e social a criança, e atendendo as necessidades da família.

\section{3 metodologia}

Para o desenvolvimento do presente estudo foi realizada uma pesquisa qualitativa bibliográfica, fundamentada em uma ampla revisão da literatura científica sobre temas relacionados a oncologia, tratamento fisioterapêutico e os métodos paliativos, sendo que os descritores de saúde utilizados foram: câncer, câncer infantil, fisioterapia, oncologia, cuidados paliativos. Os artigos foram explorados e sintetizados de forma reflexiva a fim de obter informações coerentes.

Foram pesquisados artigos em bases de dados nacionais como a Biblioteca 
Virtual da Saúde, SciELO (ScientificElectronic Library Online), LILACS (Literatura Latino Americana e do Caribe em Ciências da Saúde), google acadêmico, sites do google e PubMed. Além dessas, também foi consultado do material impresso na biblioteca da Faculdade Presidente Antônio Carlos de Teófilo Otoni e acervo pessoal, revistas e livros, dentre outras fontes de pesquisa.

Para selecionar o material pertinente, seguiram-se os seguintes passos: leitura exploratória dos textos encontrados, leitura aprofundada das obras de interesse e finalmente a leitura analítica dos trabalhos mais relevantes

\section{Discussão de resultados}

\subsection{Tratamento fisioterapêutico}

O cuidado paliativo com a criança no tratamento do câncer exige do fisioterapeuta uma atenção difusa e parâmetros metodológicos específicos no uso de recursos didáticos apropriados em ambientes hospitalares, onde as demandas embora tenham o mesmo diagnóstico, são variadas sabendo que cada criança reage de modo diferente a doença, e evidentemente ao tratamento.

A avaliação na intervenção é de grande importância, e precisa conter informações especificas e detalhadas para precauções, como história do paciente, força muscular, postura, dor (usando métodos para a avaliação de acordo com a faixa etária), avaliação de tônus muscular e resistência cardiovascular.

A criança diagnosticada com câncer está sujeita a perder o ânimo e a vontade de brincar livremente observando os aspectos do tratamento, principalmente aquelas que passaram por procedimentos cirúrgicos. Diante disso, o tratamento deve incluir o lúdico, como forma atenuante nesse processo de hospitalização, proporcionando um ambiente menos traumatizante e mais humanizado, o que possibilita e favorece a sociabilidade, interação e dinamismo mesmo com a restrição do espaço físico e de todas limitações provenientes da enfermidade afirma Silva (2006, p.2068).

Para cada sintoma há tratamentos específicos. No caso da dor, o fisioterapeuta pode recorrer a condutas como eletroterapia, crioterapia, termoterapia, e terapia manual, podendo ser utilizada também para relaxamento do paciente em 
caso de estresse, ganho de amplitude de movimento, e melhora da capacidade funcional.

Em sintomas como encurtamentos, fadiga, fraqueza muscular, úlceras de decúbitos, o profissional pode trabalhar com alongamentos, posicionamento, exercícios ativos e atividades funcionais. No caso de disfunções pulmonares e neurológicas como atelectasia, dispneia, parestesia e plegias, é usado o treino sensitivo, fortalecimento de períneo, estimulação da tosse, manobras de reexpansão pulmonar e aspiração.

Deve-se ressaltar que o caráter preventivo é um dos aspetos fundamentais dos cuidados paliativos. Antecipar possíveis complicações é da responsabilidade de todos os profissionais envolvidos, implementando medidas preventivas necessárias e aconselhando os pacientes e familiares de forma a evitar sofrimentos desnecessários (Marcucci, 2005, p.72).

\section{Conclusão}

Desde 2009, a fisioterapia oncológica é uma das áreas que apresentou um crescimento significativo, desde a sua concepção, mas mesmo com esse aumento, ainda se observar que em determinados locais não tem o reconhecimento de fisioterapeutas em equipes que atuam no tratamento do paciente com câncer.

Pelas analises bibliográficas estudadas e pesquisadas, pode-se observar que a fisioterapia desempenha um importante papel no tratamento do câncer, através dos cuidados paliativos.

Os profissionais inseridos nesse contexto, dispõe de conhecimentos e habilidades inerentes para aplicar métodos e recursos, necessários para amenizar os transtornos do câncer, sobretudo na dor e na imobilidade, que poder ser constatado nos artigos como principais disfunções dessa classe patológica.

Esses recursos e métodos são de suma importância e de muita utilidade para os cuidados paliativos, sendo que os mesmos trazem resultados comprovadamente satisfatórios para tanto a equipe de saúde, pacientes e familiares e a sua atuação de colaboração se faz positivamente, com o tratamento multiprofissional e integrado parra atendimentos dos pacientes oncológicos. 
Enfim, os estudos encontrados mostram que a fisioterapia tem um papel relevante nos cuidados paliativos, entretanto necessita-se de uma maior oferta e implementação deste tratamento nos serviços de saúde.

\section{Referências}

ARRAIS, R.C.S Atuação da fisioterapia nos cuidados paliativos oncológicos. Fisioterapeuta especialista em fisioterapia na saúde da mulher.

Unicamp/SP.[S.n.t], 2013.

Brasil, Associação Brasileira do Câncer (2007). Sobre o Câncer. Acesso em 19/06/2007. Disponível em: http://www.abcancer.org.br/sobre.php?c=8\&s= 18\&lang $=16$

Brasil, Instituto Nacional de Câncer (2007). Particularidades do câncer infantil. Acesso em 11/01/2007. Disponível em: http://www.inca.gov.br/conteudoview.asp? $\mathrm{id}=343$

CARVALHO, RT; PARSONS HÁ, Organizadores. Manual de Cuidados Paliativos ANCP: Ampliado e Atualizado. $2^{\circ}$. ed. Porto Alegre: Academia Nacional de Cuidados Paliativos; 2012.

COSTA, R. P.; Técnicas e recursos para remoção de secreção brônquica. In: SARMENTO, G. J. V. Fisioterapia respiratória no paciente crítico: rotinas clínicas. 3. ed. São Paulo: Manole, 2010.

Dávila, L. F. C. (2006). El duelo del paciente infantil con cáncer. Acesso em 12/01/2007. Disponível em http://www.psicooncologia.org/articulos//articulos_detalle. cfm?estado=ver\&id $=83 \& \mathrm{x}=91 \& \mathrm{y}=7$

GIRÃO, M.; ALVES. S. Fisioterapia nos cuidados paliativos. Revista de ciências da saúde da ESSCVP, v. 5, n.13, p. 34-41, 2013. 
GUEDES T.C. O papel da fisioterapia nos cuidados paliativos da criança com câncer. Salvador, 2015.

Gutierrez, PR. O que é o paciente terminal? Rev. Assoc. Med. Bras. 2001; 47.

Laakson, L. The role of physiotherapy in palliative care. Australian Family Physician, 2006. 35 .

MARCUCCI, F.C.I. O papel da fisioterapia nos cuidados paliativos a pacientes com câncer. Rev. Bras. Cancerol., v.51, p.67-77, 2005.

MELO, T.P.T. et al. A percepção dos pacientes portadores de neoplasia pulmonar avançada diante dos cuidados paliativos da fisioterapia. 2013. Disponível em: Acesso em: 14 set. 2016.

MULLER A.M, et al. Paciente Oncológico em Fase Terminal: Percepção e Abordagem do Fisioterapeuta. Revista Brasileira de Cancerologia 2011; 57(2): 207215.

MÜLLER, A.M.; SCORTEGAGNA, D.; MOUSSALLE, L.D. Paciente oncológico em fase terminal: percepção e abordagem do fisioterapeuta. Rev. Bras. Cancerol., v.57, n.2, p.207-215, 2011.

PANNO, J. Câncer: The Role of Genes, Lifestyle, and Environment. Nova York, 2005 PESSINI, L.; BERTACHINI, L. Humanização e cuidados paliativos. São Paulo: Loyola, 2004.

PORTO, A.R. et al. A essência da prática interdisciplinar no cuidado paliativo às pessoas com cancer. Invest. Educ. Enferm., v.30, n.2, p.231-239, 2012.

SANTOS, FS, Editor. Cuidados Paliativos: Diretrizes, Humanização e Alívio dos Sintomas. São Paulo: Atheneu; 2011. 